\title{
Impact of the Secondary Service Transmit Power Constraint on the Achievable Capacity of Spectrum Sharing in Rayleigh Fading Environment
}

\author{
Mohammad G. Khoshkholgh, Keivan Navaie, Member, IEEE, and Halim Yanikomeroglu, Member, IEEE
}

\begin{abstract}
The two main constraints on the transmit power allocation of the secondary service in a spectrum sharing scheme are the received interference threshold at the primary receiver, and the maximum transmit power of the secondary user. We obtain a critical system parameter which relates these two constraints and enables the system designer to eliminate the interference threshold constraint by adjusting the maximum transmit power of the secondary users. Eliminating the interference threshold constraint significantly reduces the system complexity by making the power allocation of the secondary service independent from the channel state information between the secondary transmitter and the primary receiver; thus removes the need for signaling between primary and secondary systems.
\end{abstract}

Index Terms-Achievable capacity, interference threshold, opportunistic spectrum access, spectrum sharing.

\section{INTRODUCTION}

$\mathbf{I}$ N SPECTRUM SHARING a Secondary Service is able to access the white spaces in a frequency band which is formally allocated to the Primary Service [1]. White spaces are those parts of the spectrum allocated to the primary user which are under-utilized in some particular times and specific locations. Various schemes are proposed in the literature for spectrum sharing (see e.g., [1] and references therein). In this letter, our focus is the Opportunistic Spectrum Access (OSA).

In this paper, we consider a DS-CDMA/OFDM spectrum sharing system in which the spectrum of a DS-CDMA-based primary service is shared with a secondary service that utilizes OFDM. DS-CDMA is the dominant air interface technique for the third generation $(3 \mathrm{G})$ mobile communications (e.g., UMTS, and cdma2000) and some Wireless Local Area Network (WLAN) technologies, (e.g., IEEE 802.11b). Therefore, the spectrum sharing over existing DS-CDMA-based networks is anticipated to be one of the spectrum sharing applications in the near future. On one hand, OFDM provides the required flexibility to the secondary service to access separate underutilized portions of the spectrum band. On the other hand, the spreading characteristic of DS-CDMA makes it more robust to narrow-band interference created by OFDM secondary users.

DS-CDMA systems are interference-limited [2]. In such systems as a metric for recognizing a white space here we consider a threshold on the acceptable level of the imposed interference at the primary receiver caused by the operation

Manuscript received July 21, 2008. The associate editor coordinating the review of this letter and approving it for publication was G. Mazzini.

M. G.Khoshkholgh and K. Navaie are with the ECE Department, Tarbiat Modares University, Tehran, Iran.

H. Yanikomeroglu is with the SCE Department, Carleton University, Ottawa, ON, Canada (e-mail: \{keivan, halim\}@sce.carleton.ca).

Digital Object Identifier 10.1109/LCOMM.2008.081153 of the secondary user. Therefore, a white space is defined as a frequency band in which the interference level is below the Interference Threshold.

To achieve the maximum capacity in OSA, the secondary users should adjust their transmit powers over the white spaces. Two main constraints affect the secondary users transmit power: the received interference at the primary receiver, and the maximum transmission power of the secondary users.

To obtain the maximum achievable capacity in [3], the interference threshold constraint is considered as the dominant constraint; thus, the secondary service system constraint on the maximum transmit power is simply ignored. Since this assumption may result in allocating a transmit power level to a secondary user which is higher than its maximum transmit power, it results in an upper bound to the achievable capacity.

In this letter we show that these two constraints can be considered as the system design tools through carefully selecting the system parameters. Particularly, we show that by adjusting the secondary service maximum transmit power we are able to eliminate the interference threshold constraint. This result is very important in practice since the interference threshold constraint should be satisfied at the receiver of the primary service, and examining this constraint imposes an inter-system signaling overhead, thus increases the system complexity.

\section{SySTEM MODEL}

We consider a frequency-selective block fading wireless channel with AWGN. The coherence bandwidth of the channel is $B_{c} \mathrm{~Hz}$. The white noise power spectral density is $N_{0}$. The channel is divided into $N$ flat fading $B_{c} \mathrm{~Hz}$ sub-channels. Sub-channels are indexed by $i=1,2, \ldots, N$. Based on the primary network status, the secondary service may have access to $M$ accessible sub-channels indexed by $j=0,1, \ldots, M$ where $0 \leq M \leq N$ i.e., OFDM. The interference threshold, $Q$, is the maximum allowable average interference at the receiver of the primary service caused by the operation of the secondary service in the spectrum. Since our focus is on the secondary service maximum achievable capacity, similar to the spectrum sharing literature (see e.g., [3], [4]), we consider only one secondary user. In cases where more than one secondary users are competing for accessing the white spaces, due to probable inter-secondary users interference because of the imperfectness in multiple access techniques, the total secondary service achievable capacity is upper-bounded by the case with only one secondary user.

For sub-channel $i, g_{0 i}$ and $g_{1 i}$ denote the instantaneous power gains of sub-channel $i$ from the secondary transmitter to the primary and the secondary receivers, respectively. Both $g_{0 i}$ 
and $g_{1 i}$ are assumed to be stationary and ergodic independent random variables with unit-mean probability density functions (pdf), $f_{0 i}\left(g_{0 i}\right)$, and $f_{1 i}\left(g_{1 i}\right)$, respectively, which are independent and identically distributed (i.i.d.) for $i=1,2, \ldots, N$. Generally, the instantaneous transmit power of the secondary transmitter in the $i$ th sub-channel is $P_{s i}$ which should be carefully obtained based on the system constraints; thus is a function of $g_{0 i}$, and $g_{1 i}$, i.e., $P_{s i}\left(g_{0 i}, g_{1 i}\right)$. For brevity, we refer to $P_{s i}\left(g_{0 i}, g_{1 i}\right)$ as $P_{s i}$. The maximum transmit power of the secondary transmitter is $\bar{P}_{s}$.

In DS-CDMA networks with large enough number of users, it is shown in the literature (see e.g., [2]) that the total received interference caused by other active users in the network coverage area is simply modelled as an additive white Gaussian noise with a power spectral density equal to the product of the background noise power spectral density and a factor $(K-1)$. Since the secondary user is OFDM, the interference received from the primary service in each $B_{c} \mathrm{~Hz}$ sub-channel can be modelled as an additive white Gaussian noise with power spectral density of $(K-1) N_{0} B_{c}, K \geq 1$.

\section{MaXimum Achievable Capacity}

Assume that there are $M$ accessible sub-channels for the secondary service. In practice $M$ can be assigned by the regulator or the primary service operator. The maximum achievable capacity of the secondary service over $M$ accessible subchannels is the solution of the following optimization problem:

$$
\begin{aligned}
C_{s \mid M}=\max _{\mathbf{P}_{s}} & \sum_{j=1}^{M} B_{c} \mathbb{E}_{g_{1 j}} \mathbb{E}_{g_{0 j}}\left[\log \left(1+\frac{g_{1 j} P_{s j}}{K N_{0} B_{c}}\right)\right], \\
\text { s.t. } & \sum_{j=1}^{M} \mathbb{E}_{g_{1 j}} \mathbb{E}_{g_{0 j}} P_{s j} \leq \bar{P}_{s} \\
& \sum_{j=1}^{M} \mathbb{E}_{g_{1 j}} \mathbb{E}_{g_{0 j}} g_{0 j} P_{s j} \leq G Q .
\end{aligned}
$$

where $\mathbb{E}_{x}\{h(x)\} \triangleq \int_{x} h(x) f_{X}(x) d x, f_{X}(x)$ is the pdf of random variable $X, \mathbf{P}_{s}=\left(P_{s 1}\left(g_{01}, g_{11}\right), \ldots, P_{s M}\left(g_{0 M}, g_{1 M}\right)\right)$, $G$ is the processing gain of DS-CDMA primary network, and $Q$ is the interference threshold. In the above formulation, the objective function of the optimization problem, (1), is Shannon's Ergodic capacity averaged over both $g_{0 j}$ and $g_{1 j}$ and aggregated for all accessible sub-channels. Equation (2) is the transmit power constraint for the secondary service and (3) is the primary service interference constraint. In (3) the secondary service's operation in each sub-channel acts as a narrow-band interference after being multiplied by $1 / G$ due to the spreading behavior [2] of DS-CDMA systems. In the above, $\bar{P}_{s}, Q$, and $G$ are the main system parameters.

The optimization problem in (1) can be solved using Lagrange Multipliers method. Note that constraint (2) can be examined in the secondary service transmitter and it only requires the channel state information between the secondary transmitter and receiver. However, the constraint in (3) requires the channel state information between the secondary transmitter and the primary receiver. Providing such information to the secondary transmitter imposes a system overhead. Therefore, finding the optimal power allocation $\mathbf{P}_{s}^{*}$ in (1) is very complex.
In practice, we can recognize two following cases. First, the case where $\bar{P}_{s}$ is very high (i.e., there is no practical power constraint for the secondary service), and/or the interference threshold $Q$ is small enough, so that for any feasible power allocation holds in (3), the power constraint in (2) is always satisfied. In this case, the dominant constraint is the interference threshold constraint in (2). This assumption is usually made in the literature to find the maximum achievable capacity of the secondary service (see e.g., [3]). We refer to this case as the Interference Constrained spectrum sharing.

The second case is the one where the interference threshold $Q$ is very high, and/or $\bar{P}_{s}$ is small enough, so that the corresponding created interference at the front end of the primary service receiver never crosses the $Q$. In such cases, the interference constraint in (3) is always satisfied. Thus, the dominant constraint is the maximum transmit power constraint of the secondary service in (2). We refer to this case as Power Constrained spectrum sharing.

\section{A. Interference Constrained Spectrum Sharing}

In the interference constrained spectrum sharing, the optimization problem in (1) is simplified to

$$
\begin{aligned}
C_{s \mid M}^{I}= & \max _{\mathbf{P}_{s}} \sum_{j=1}^{M} B_{c} \mathbb{E}_{g_{1 j}} \mathbb{E}_{g_{0 j}}\left[\log \left(1+\frac{g_{1 j} P_{s j}}{K N_{0} B_{c}}\right)\right], \\
& \text { s.t. } \sum_{j=1}^{M} \mathbb{E}_{g_{1 j}} \mathbb{E}_{g_{0 j}} g_{0 j} P_{s j} \leq G Q .
\end{aligned}
$$

By utilizing Lagrange Multipliers method, the optimal transmission power is obtained as $P_{s j}^{*}=\left(\frac{1}{\lambda_{1}^{*} g_{0 j}}-\frac{K N_{0} B_{c}}{g_{1 j}}\right)^{+}$, where $(x)^{+}=\max \{0, x\}$, and $\lambda_{1}^{*}$ is Lagrangian coefficient. For Rayleigh fading, (5) is simplified as

$$
\sum_{j=1}^{M} \int_{\frac{g_{1 j}}{g_{0 j}} \geq \rho_{1}}\left(\frac{1}{\rho_{1}}-\frac{g_{0 j}}{g_{1 j}}\right) e^{-g_{1 j}} e^{-g_{0 j}} d g_{0 j} d g_{1 j}=\gamma_{Q},
$$

where $\rho_{1} \triangleq \lambda_{1}^{*} K N_{0} B_{c}$ and $\gamma_{Q} \triangleq G Q\left(K N_{0} B_{c}\right)^{-1}$.

In the interference constrained spectrum sharing, $\gamma_{Q}$ is the ratio of the maximum allowable average interference cause by the secondary service, i.e., $G Q$, to the total interference-plusnoise over one sub-channel in DS-CDMA primary network, i.e., $K N_{0} B_{c}$. A higher $\gamma_{Q}$ results in a higher achievable capacity of the secondary service.

We define $\nu_{j} \triangleq g_{1 j} g_{0 j}^{-1}$. For i.i.d. Rayleigh random variables $g_{0 j}$ and $g_{1 j}$, the pdf of $\nu_{j}, h\left(\nu_{j}\right)$, is obtained through integrating by parts as follows:

$$
h\left(\nu_{j}\right)=\frac{d}{d \nu_{j}} \int_{0}^{\infty} \int_{0}^{g_{0 j} \nu_{j}} e^{-g_{0 j}} e^{-g_{1 j}} d g_{0 j} d g_{1 j}=\frac{1}{\left(1+\nu_{j}\right)^{2}} .
$$

Following the same line of argument as above, it is simple to show that the random variable $1 / \nu_{j}$ has the same pdf. Therefore, (6) is simplified as

$$
\rho_{1}-\log \left(1+\rho_{1}\right)=\frac{\gamma_{Q}}{M} .
$$




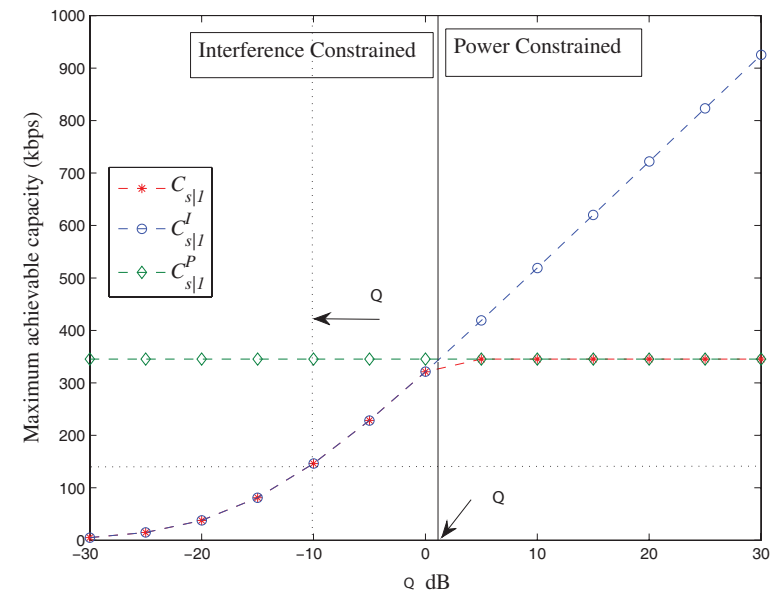

Fig. 1. The maximum achievable capacity of the secondary service for $\bar{P}_{s}=1$ Watt, $K=2, B_{c}=60 \mathrm{KHz}$, and $G=125$.

Substituting the obtained $P_{s j}^{*}$ into (4), the maximum achievable capacity for interference constrained spectrum sharing is

$$
C_{s \mid M}^{I}=M B_{c} \log \left(1+\rho_{1}\right)
$$

where $\rho_{1}$ is calculated from (8).

\section{B. Power Constrained Spectrum Sharing}

In the power constrained spectrum sharing the interference threshold is always satisfied. Therefore, the allocated power to the secondary user is not a function of the channel gain between the primary receiver and the secondary transmitter, $g_{0 j}$, thus is evaluated only based on $g_{1 j}$. Consequently, the optimization problem in (1) is simplified as

$$
\begin{aligned}
C_{s \mid M}^{P}= & \max _{\mathbf{P}_{s}} \sum_{j=1}^{M} B_{c} \mathbb{E}_{g_{1 j}}\left[\log \left(1+\frac{g_{1 j} P_{s j}}{K N_{0} B_{c}}\right)\right], \\
\text { s.t. } & \sum_{j=1}^{M} \mathbb{E}_{g_{1 j}} P_{s j} \leq \bar{P}_{s} .
\end{aligned}
$$

By utilizing Lagrange Multipliers method, the optimal transmit power is obtained as $P_{s j}^{*}=\left(\frac{1}{\lambda_{2}^{*}}-\frac{K N_{0} B_{c}}{g_{1 j}}\right)^{+}$, where $\lambda_{2}^{*}$ is Lagrangian coefficient. For Rayleigh fading, the constraint in (11) is simplified as

$$
\sum_{j=1}^{M} \int_{g_{1 j} \geq \rho_{2}}\left(\frac{1}{\rho_{2}}-\frac{1}{g_{1 j}}\right) e^{-g_{1 j}} d g_{1 j}=\gamma_{P}
$$

where $\rho_{2} \triangleq \lambda_{2}^{*} K N_{0} B_{c}$ and $\gamma_{P} \triangleq \bar{P}_{s}\left(K N_{0} B_{c}\right)^{-1}$.

In the power constrained spectrum sharing, $\gamma_{P}$ is the ratio of the maximum transmit power of the secondary service, i.e., $\bar{P}_{s}$, to the total interference-plus-noise in DS-CDMA primary network over one sub-channel, i.e., $K N_{0} B_{c}$. Conceptually, $\gamma_{P}$ in power constrained spectrum sharing is corresponding to $\gamma_{Q}$ in interference constrained spectrum sharing. A higher $\gamma_{P}$ results in a higher secondary service achievable capacity.

Using (12), $\rho_{2}$ is obtained from the following equation:

$$
\frac{e^{-\rho_{2}}}{\rho_{2}}-E_{1}\left(\rho_{2}\right)=\frac{\gamma_{P}}{M},
$$

where $E_{n}(x)$ is the exponential integral of order $n$ defined as $E_{n}(x) \triangleq \int_{1}^{\infty} t^{-n} e^{-x t} d t, x \geq 0$. Substituting the obtained $P_{s j}^{*}$ in this case into (10), the maximum achievable capacity is

$$
C_{s \mid M}^{P}=\sum_{j=1}^{M} \int_{g_{1 j} \geq \rho_{2}} B_{c} \log \left(\frac{g_{1 j}}{\rho_{2}}\right) e^{-g_{1 j}} d g_{1 j}=M B_{c} \rho_{2} J_{1}\left(\rho_{2}\right),
$$

where $\quad J_{n}(x) \triangleq \int_{1}^{\infty} t^{n-1} e^{-x t} \log (t) d t, \quad x \geq 0 . \quad$ Noting $E_{1}(x)=x J_{1}(x),(14)$ is reduced to

$$
C_{s \mid M}^{P}=M B_{c} E_{1}\left(\rho_{2}\right) .
$$

\section{Impact of Power and Interference Constraints}

To study the impact of the constraints (2) and (3), we compare $C_{s \mid M}, C_{s \mid M}^{P}$, and $C_{s \mid M}^{I}$. In Fig. $1, C_{s \mid 1}, C_{s \mid 1}^{P}$, and $C_{s \mid 1}^{I}$ are shown versus $\gamma_{Q}$ for $\bar{P}_{s}=1$ Watt. As it is seen, for a given $\bar{P}_{s}$ there is a threshold $\gamma_{Q}^{*}$ such that for $\gamma_{Q}<\gamma_{Q}^{*}$ the system is well approximated by an interference constrained spectrum sharing system, and $C_{s \mid 1} \approx C_{s \mid 1}^{I}$. It is further seen that the system behavior for $\gamma_{Q}>\gamma_{Q}^{*}$ can be well approximated by a power constrained spectrum sharing system, and $C_{s \mid 1} \approx C_{s \mid 1}^{P}$.

As it is seen in the above discussion, $\gamma_{Q}^{*}$ is a critical system parameter where $C_{s \mid M}^{I}$ crosses $C_{s \mid M}^{P}$. By considering (8), (9), (13), and (15), $\gamma_{Q}^{*}$ for Rayleigh fading channels is obtained as

$$
\gamma_{Q}^{*}=\gamma_{P}+M\left(\rho_{1}-\frac{e^{-\rho_{2}}}{\rho_{2}}\right) .
$$

As it is observed in Fig. 1, for a given interference threshold $Q$ (or $\gamma_{Q}$ ) which is the system operation point, based on (16) by adjusting the maximum transmit power, $\bar{P}_{s}$ (or $\gamma_{P}$ ), it is possible to transform an interference constrained system to a power constrained system. In other words, by adjusting $\bar{P}_{s}$ one can move $\gamma_{Q}^{*}$ to the left hand side of the actual system operation point, $\gamma_{Q}$, and make the system act as a power constrained system. Such transformation removes the interference constraint (3); thus eliminates the need for the channel state information between the secondary transmitter and the primary receiver (i.e., $g_{0 i}, i=1, \ldots, M$ ). Therefore, such transformation significantly reduces the system complexity. As it is observed in Fig. 1, by considering an appropriate $\bar{P}_{s}$ so that the new $\gamma_{Q}^{*}$ is smaller than or equal to $\gamma_{Q}$ this transformation does not affect the maximum achievable capacity of the secondary service.

\section{REFERENCES}

[1] Q. Zhao and B. Sadler, "A survey of dynamic spectrum access: Signal processing, networking, and regulatory policy," IEEE Signal Processing Mag., vol. 24, no. 3, pp. 79-89, May 2007.

[2] A. J. Viterbi, CDMA: Principles of Spread Spectrum Communication. Addison-Wesley, 1995.

[3] A. Ghasemi and E. S. Sousa, "Fundamental limits of spectrum-sharing in fading environments," IEEE Trans. Wireless Commun., vol. 6, no. 2, pp. 649-658, Feb. 2007.

[4] S. A. Jafar and S. Srinivasa, "Capacity limits of cognitive radio with distributed and dynamic spectral activity," IEEE J. Select. Areas Commun., vol. 25, no. 3, pp. 529-537, Apr. 2007. 